\title{
Saturated Power Control Scheme for Kalman Filtering via Wireless Sensor Networks*
}

\author{
Dongmei Zhang', Zhuangwei Miao', Xingang Wang ${ }^{2}$ \\ ${ }^{1}$ College of Science, Zhejiang University of Technology, Zhejiang, China \\ ${ }^{2}$ College of Information Engineering, Zhejiang, China \\ Email: meidzh@zjut.edu.cn,miao.z.w@zjut.edu.cn,wxg@zjut.edu.cn
}

Received August 12, 2013; revised September 12, 2013; accepted September 19, 2013

Copyright (C 2013 Dongmei Zhang et al. This is an open access article distributed under the Creative Commons Attribution License, which permits unrestricted use, distribution, and reproduction in any medium, provided the original work is properly cited.

\begin{abstract}
We investigate the Kalman filtering problem via wireless sensor networks over fading channels. When part or all of the observation measurements are lost in a random fashion, we obtain the conclusion that the packet dropout probabilities depend upon the time-varying channel gains and the transmission power levels used by the sensors. We develop a saturated power controller which trades off sensor energy expenditure versus state estimation accuracy. The latter is measured by the expected value of the future covariance matrices provided by the associated time-varying Kalman filter. We study the statistical convergence properties of the error covariance matrix and pointed out the existence of the admissible packet arrival probability bound.
\end{abstract}

Keywords: Power Control; Kalman Filtering; Fading Channel; Packet Dropout

\section{Introduction}

Recently, the rapid evolution of wireless sensor networks, see e.g., [1-4] has made wireless sensors (WSs) cheap and reliable enough to be brought into commercial use. WSs offers several advantages for industrial control systems, such as flexibility, low cost, and fast deployment. Furthermore, WSs and actuators can be placed where wires cannot go, or where power sockets are unavailable. The use of wireless communications for state estimation and closed loop control, however, also brings new challenges in system design.

A drawback of using wireless channels is that they are subject to fading and interference, which frequently leads to packet errors [5-8]. Interestingly, the time-variability of the fading channel can be compensated for by adjusting the power levels [9-13]. Since energy is severely limited in most WS applications, power control design involves trading energy consumption for accuracy [14-18]. Thus, an important challenge is to design suitable power control schemes [19].

Kalman filters are widespread in estimation and control applications, and the effect of random measurement losses on filter stability has received considerable attention in the literature. In particular, Sinopoli et al. [20]

*This work is supported by National Natural Science Foundation (NNSF) of China under Grant 61074039, 61273116 and 1101367. studied a single link estimation setup for linear timeinvariant (LTI) plants where the packet dropout processes are independent and identically distributed (i.i.d.). In this modeling paradigm, it was assumed that the packet dropout probability remains unchanged from one transmission to the next, and all the sensor measurements need to be encoded in a single packet and the sensors should be collocated. The result was extended to deal with the general case of multi-channel Kalman filtering problem [21]. The existence of the critical arrival rate of the observation was proved and both the upper and the lower bound were computed. Plarre and Bullo [22] examines a state estimation architecture with two channels affected by i.i.d. packet dropouts. The case of Kalman filtering with a single link was investigated in Huang and Dey [23-25], where the packet dropouts are described by a time homogeneous two-state Markov chain. Zhang [26] considered a multi-rate distributed estimation problem for wireless sensor networks with packet dropout, the output measurements of the adjacent nodes' are utilized to generate the state estimation and then fused. In [27], sufficient conditions are established which ensure the convergence of the Kalman filter co-variance matrix. The conditions obtained are used to formulate stabilizing optimal power allocation laws which minimize the total power used by each sensor.

Inspired by the previous work [27], saturated power 
controllers are designed which provide a time-varying successful probability by inverting the channel gain. We can prove that there exists a critical value such that the expectation of the estimation error covariance is always bounded if the arrival probability of an observation at time is greater than the value. We call the critical value as the admissible packet arrival rate and the computational procedure is presented to design the power controller gain according to the value.

Notation: We write $\operatorname{Pr}(\Omega \mid \Delta)$ for the conditional probability of $\Omega$ given $\Delta$, and $\operatorname{Pr}(\Omega)$ for the unconditional probability. A random vector $v$, which is Gaussian distributed with mean value $m$ and covariance matrix $\Gamma>0$ is denoted by $v \sim N(m, \Gamma)$.

\section{Problem Formulation}

Consider an uncontrolled LTI $n$-dimensional systems:

$$
x_{k+1}=A x_{k}+w_{k}
$$

where the initial system state is Gaussian distributed with mean $x_{0}$ and covariance $P_{0}$. The driving noise process $w_{k}, k \in N$ is i.i.d., where each $w_{k} \sim N(0, Q)$.

To remotely estimate the system state sequence $x=x_{k}(k \in N)$, a network of $L$ sensors is used. Each sensor $l(\in\{1,2, \cdots, L\})$ provides a nosiy measurement signal, say $y_{l}=y_{l, k}(k \in N)$ :

$$
y_{l, k}=C_{l} x_{k}+v_{l, k}
$$

where $v_{l}=v_{l, k}(k \in N)$ is an i.i.d. process with each $v_{l, k} \sim N\left(0, R_{l}\right)$

The $L$ values in (2) are transmitted through the wireless links to the single gateway. The received signals are then used to remotely estimate the state of system (1). In the following scenario, we assume that $L=2$ for the sake of convenience, i.e., there are two measurement outputs encoded separately and sent over different wireless channels at each time step:

$$
y_{k}=\left[\begin{array}{c}
y_{1, k} \\
v_{2 . k}
\end{array}\right]=\left[\begin{array}{l}
C_{1} \\
C_{1}
\end{array}\right] x_{k}+\left[\begin{array}{c}
v_{1, k} \\
v_{2, k}
\end{array}\right]
$$

with $R=\operatorname{cov}\left(v_{1, k}, v_{2, k}\right)=\left[\begin{array}{l}R_{1}, 0 \\ 0, R_{2}\end{array}\right]$.

\subsection{Channel with Rayleigh Fading}

In the present work, we will adopt a block-fading channel gain model, which is a common information theoretic model for fading wireless channels where the channel power gains remain invariant over a block [21]. Furthermore, we assume the single measurement $y_{l}(k)$ to be a packet, thus, the block length equals the packet transmission time. The associated channel gain processes are denoted by $h_{l}=h_{l, k}$ (" $k \in N$ ”), which are exponentially dis- tributed with cdf

$$
F_{h_{l, k}(h)}= \begin{cases}1-e^{-\lambda_{l} h_{l}} & \text { if } h_{l} \geq 0 \\ 0 & \text { if } h_{l}<0\end{cases}
$$

where, $\lambda_{l}>0$.

Suppose that the binary phase shift keying transmission over the i.i.d. block fading additive white Gaussian noise channels is used [26]. Each sensor measurement consists of a packet containing $b$ bits. Since the bit errors are independent within a packet, the success probabilities can be written as, see [6],

$$
f_{l}(h u)=\left(\int_{0}^{\sqrt{h u}} \frac{1}{\sqrt{2 \pi}} e^{-t^{2} / 2} \mathrm{~d} t\right)^{b}
$$

After approximate calculation, we have the following theorem.

Theorem 1

$$
f_{l}(h u) \doteq\left(1-e^{\frac{2}{\pi} h u}\right)^{\frac{b}{2}}
$$

Proof: For a random variable $X \sim N(0,1)$, its cdf can be represented by

$$
\Phi_{X}(x)=\int_{-\infty}^{x} \frac{1}{\sqrt{2 \pi}} e^{-\frac{t^{2}}{2}} \mathrm{~d} t
$$

It is easy to see

$$
\begin{aligned}
{[\Phi(a)-\Phi(-a)]^{2} } & =\frac{1}{2 \pi} \int_{-a}^{a} \int_{-a}^{a} e^{-\frac{x^{2}+y^{2}}{2}} d x d y \\
& =\frac{1}{2 \pi} \int_{0}^{2 \pi} \int_{0}^{R} e^{-\frac{\rho^{2}}{2}} \rho d \rho d \theta \\
& =1-e^{-\frac{1}{2} R^{2}}
\end{aligned}
$$

The second equality is obtained if we approximate square with round, i.e.,

$$
\pi R^{2}=4 a^{2}
$$

and obtain

$$
\Phi(a)=\frac{1}{2}\left(1 \pm \sqrt{1-e^{-\frac{2}{\pi} a^{2}}}\right)
$$

which yields (5). This ends the proof of Theorem 1.

Since the $l$ links between sensors and gateway are wireless, transmission errors are likely to occur. Faulty packets will be discarded when estimating the system state. We will model transmission effects by introducing the $l$ binary stochastic arrival processes $\gamma_{l}=\gamma_{l, k}(k \in N)$, where:

$$
\gamma_{l, k}=\left\{\begin{array}{l}
1 \text { if } y_{l, k} \text { arrives error-free at time } k \\
0 \text { if } y_{l, k} \text { arrives error-free at time } k
\end{array}\right.
$$

The success probabilities of $\gamma_{l, k}$ are determined by the propagation environment and transmission power. In 
the present work, the saturated power controller is used

$$
u_{l, k}= \begin{cases}K_{l} / h_{l, k} & \text { if } h_{l, k} \geq K_{l} / u_{l}^{\max } \\ u_{l}^{\max } & \text { if } h_{l, k}<K_{l} / u_{l}^{\max }\end{cases}
$$

where $u_{l}^{\max }$ is the peak power level, and $K_{l}$ is the power controller gain for the $l$ channel. Moreover,

$$
\operatorname{Pr}\left(\gamma_{l, k}=1 \mid h_{l, k}=h, u_{l, k}=u\right)=f_{l}(h u)
$$

For further reference, we also define the overall arrival process of all $l$ links, say $\gamma_{l}=\gamma_{l}(k)_{k \in N}$ via

$$
\gamma(k)=\left[\gamma_{1}(k)^{\prime}, r_{2}(k)^{\prime}\right]^{\prime}
$$

We will assume that the data transmission incorporates error detection coding. Hence, the gateway knows the packets received from the sensors contain errors or not. Thus, at any time $k$, the past and present realizations of the overall transmission process are available at the gateway. For state estimation purposes, the system amounts to sampling (1)-(2) only at the successful transmission instants of each sensor link. Indeed, the conditional probability distribution of the system state at any time $k$, given the correctly received sensor measurements up to time $k$, is Gaussian.

Let $\gamma_{0}^{k}=\{\gamma(0), \gamma(1), \cdots, \gamma(k)\}, y_{0}^{k}=\left\{y_{0}, y_{1}, \cdots, y_{k}\right\}$, we define

$$
\begin{aligned}
& \hat{x}_{k \mid k}=E\left[x_{k} \mid y_{0}^{k}, \gamma_{0}^{k}\right] \\
& P_{k \mid k}=E\left[\left(x_{k}-\hat{x}_{k \mid k}\right)\left(x_{k}-\hat{x}_{k \mid k}\right) \mid y_{0}^{k}, \gamma_{0}^{k}\right] \\
& \hat{x}_{k+1 \mid k}=E\left[x_{k+1} \mid y_{0}^{k}, \gamma_{0}^{k}\right] \\
& P_{k+1 \mid k}=E\left[\left(x_{k+1}-\hat{x}_{k+1 \mid k}\right)\left(x_{k+1}-\hat{x}_{k+1 \mid k}\right)^{\prime} \mid y_{0}^{k}, \gamma_{0}^{k}\right]
\end{aligned}
$$

The time update of the Kalman filter is independent of the observation process and thus stays the same as in the classical Kalman filter:

$$
\begin{aligned}
& \hat{x}_{k+1 \mid k}=A \hat{x}_{k \mid k} \\
& P_{k+1 \mid k}=A P_{k \mid k} A^{\prime}+Q
\end{aligned}
$$

The measurement update is now stochastic since the received measurements depend on the random variables $\gamma_{1, k}$ and $\gamma_{2, k}$.

Let $P_{k}=P_{k \mid k-1}$, a general form of the error covariance matrix is given by

$$
\begin{aligned}
P_{k+1}= & A P_{k} A^{\prime}+Q-\gamma_{1, k} \gamma_{2, k} A P_{k} C^{\prime}\left(C P_{k} C^{\prime}+R\right)^{-1} C P_{k} A^{\prime} \\
& -\gamma_{1, k}\left(1-\gamma_{2, k}\right) A P_{k} C_{1}^{\prime}\left(C_{1} P_{k} C_{1}^{\prime}+R_{1}\right)^{-1} C_{1} P_{k} A^{\prime} \\
& -\left(1+\gamma_{1, k}\right) \gamma_{2, k} A P_{k} C_{2}^{\prime}\left(C_{2} P_{k} C_{2}^{\prime}+R_{2}\right)^{-1} C_{2} P_{k} A^{\prime}
\end{aligned}
$$

In the next section, we will focus on the statistical properties of the error estimation covariance $P_{k}$.

\section{Convergence Conditions}

First, we define the modified algevraic Riccati equation for the Kalman filter with random packet dropout as follows,

$$
\begin{aligned}
g_{\lambda_{1} \lambda_{2}}(X)= & A X A^{\prime}+Q-\lambda_{1} \lambda_{2} A X C^{\prime}\left(C X C^{\prime}+R\right)^{-1} C X A^{\prime} \\
& -\lambda_{1}\left(1-\lambda_{2}\right) A X C_{1}^{\prime}\left(C_{1} X C_{1}^{\prime}+R_{1}\right)^{-1} C_{1} X A^{\prime} \\
& -\left(1+\lambda_{1}\right) \lambda_{2} A X C_{2}^{\prime}\left(C_{2} X C_{2}^{\prime}+R_{2}\right)^{-1} C_{2} X A^{\prime}
\end{aligned}
$$

Some useful properties of the function $g_{\lambda_{1} \lambda_{2}}(X)$ are presented and the proof can be found in [21].

Lemma 1 [21] $g_{\lambda_{1} \lambda_{2}}(X)$ is concanve and nondecreasing function in $X$.

Lemma 2 [21] For fixed $0<\lambda_{1}<1, g_{\lambda_{1} \lambda_{2}}(X)$ is decreasing in $\lambda_{2}$, vice versa for $\lambda_{1}$.

Lemma 3 [21] $E g_{\lambda_{1} \lambda_{2}}(X) \leq g_{\lambda_{1} \lambda_{2}}(E X)$.

Lemma 4 [21] Suppose $\exists$ matrices $\bar{K}, \bar{K}_{1}, \bar{K}_{2}$ and $\bar{P}>0$ such that $\bar{P} \geq \varphi\left(\bar{K}, \bar{K}_{1}, \bar{K}_{2}, \bar{P}\right)$, then

a) $\forall P_{0} \geq 0$, the iteration $\bar{P}_{k+1}=g_{\lambda_{1} \lambda_{2}}\left(\bar{P}_{k}\right)$ converges and

$$
\lim \operatorname{it}_{k \rightarrow \infty} g_{\lambda_{1} \lambda_{2}}^{k}\left(\bar{P}_{0}\right)=\bar{P}
$$

independent of initial condition $P_{0}$.

b) $\bar{P}$ is the unique positive semidefinite solution of $\bar{P}_{k+1}=g_{\lambda_{1} \lambda_{2}}\left(\bar{P}_{k}\right)$

Lemma 5 [21] Assuming $(A, Q)$ is controllable and $(A, C)$ observable. Fix $0 \leq \lambda_{1} \leq 1$. If $g_{\lambda_{1} \lambda_{2}}\left(P_{k}\right)$ is unstable for $\lambda_{2}=0$, whereas stable for $\lambda_{2}=1$, then $\exists \lambda_{2 c} \leq 1$ such that

$$
\lim \text { it }_{k \rightarrow \infty} E\left[P_{k}\right]=+\infty \text { for } \lambda_{2} \leq \lambda_{2 c}
$$

Furthermore, there exists a positive semidefinite matrix $M_{P_{0}}>0$ as a function of the initial condition $P_{0}>0$, such that

$$
E\left[P_{k}\right] \leq M_{P_{0}}, \forall t \text { for } \lambda_{2 c} \leq \lambda_{2} \leq 1
$$

The vice visa for fixed $\lambda_{2}$.

The following theorem gives the power controller gain region for the above critical value $\lambda_{i c}(i=1,2)$.

Theorem 2 Assuming $(A, Q)$ is controllable and $(A, C)$ observable. Fix $0 \leq \lambda_{1} \leq 1$. If $g_{\lambda_{1} \lambda_{2}}\left(P_{k}\right)$ is unstable which meets (13), then the corresponding power controller gain satisfies

$$
K_{l}>\frac{\pi}{2} \ln \left(1-\sqrt[\frac{b}{2}]{\lambda_{2 c}}\right)
$$

for $\lambda_{2 c}$ mentioned above.

Proof: The proof is established based on Theorem 1 
and Lemma 5. When $\lambda<\lambda_{2 c}$, Equation (5) has an equivalent form

$$
h_{l} u_{l}>\frac{\pi}{2} \ln \left(1-\sqrt[\frac{b}{2}]{\lambda}_{2 c}\right)
$$

when $K_{l}<h_{l} u_{l}^{\max }$, we can easily obtain

$$
K_{l}=h_{l} u_{l}>\frac{\pi}{2} \ln \left(1-\sqrt[\frac{b}{2}]{\lambda_{2 c}}\right)
$$

when $K_{l}>h_{l} u_{l}^{\max }$, we obtain $u_{l}=u_{l}^{\max }$ from (6), which yields

$$
K_{l}>h_{l} u_{l}^{\max }>h_{l} u_{l}
$$

this ends the proof of Theorem 2.

The distributed protocol mentioned above is sketched below:

\section{Procedure:}

Step 1) For given $0<\lambda_{1}<1$, we use bisection method to find $\lambda_{2 c}$. For $\forall \varepsilon>0$, search the $[0,1]$ interval to find some $\lambda_{2}^{1}$, such that

$$
\left|g_{\lambda_{1} \lambda_{2}}\left(P_{k+1}\right)-g_{\lambda_{1} \lambda_{2}}\left(P_{k}\right)\right|<\varepsilon \text {. }
$$

Step 2) For the same $\varepsilon>0$ and $0<\lambda_{1}<1$, search the $\left[\lambda_{2}^{1}, 1\right]$ interval to find some $\lambda_{2}^{2}$, such that $\left|g_{\lambda_{1} \lambda_{2}}\left(P_{k+1}\right)-g_{\lambda_{1} \lambda_{2}}\left(P_{k}\right)\right|<\varepsilon$. Use this method, we can find the greatest lower bound of the critical value $\lambda_{2 c}$.

Step 3) Design the power controller gain according to the above admissive upper bound obtained in Step 2).

Remark 1 It follows from the reasoning above that one can improve the transmission reliability and the state estimation accuracy for a given wireless propagation environment simply by increasing the power controller gain used by the transmitter. However, it is of fundamental importance to save energy in wireless sensor networks. There is the tradeoff between the stability of Kalman filter and a energy resource.

\section{Conclusions}

An energy efficient power control scheme for state estimation via wireless sensor networks operating over fading channels was presented. The time variability of the fading channels frequently leads to transmission errors with subsequent random packet drops. State estimation in the face of intermittent observations was then performed by a time-varying Kalman filter. In our scheme, the transmission power of the radio amplifier of each of the wireless sensors was controlled by the gateway. For that purpose, we proposed a saturated power controller.

The results in this paper can also be generalized to the case where the observation data is contained in more than two packets. In the generalization, the Kalman filter updating upon one or more packet losses can be computed in the same manner since we can always divide the observation into two parts: the received and the lost. The statistical properties of the error covariance matrix iteration stay the same with appropriate extensions. In our future work, we would like to study the convergence properties of the Kalman filtering problem.

\section{REFERENCES}

[1] H. Gharavi and S. P. Kumar, "Special Section on Sensor Networks Andapplications," Proceedings of the IEEE, Vol. 91, No. 8, 2003, pp. 1151-1152. http://dx.doi.org/10.1109/JPROC.2003.814925

[2] P. Havinga, J. C. Hou and F. Zhao, "Wireless Sensor Networks,” IEEE Wireless Communications, Vol. 11, No. 12, 2004, pp. 4-5.

[3] M. Ilyas, I. Mahgoub and L. Kelly, "Handbook of Sensor Networks: Compact Wireless and Wired Sensing Systems,” CRC-Press, Boca Raton, 2004. http://dx.doi.org/10.1201/9780203489635

[4] X. Shen, Q. Zhang and Q. M. Cai, "Wireless Sensor Networking,” IEEE Wireless Communications, Vol. 14, No. 6, 2007, pp. 4-5. http://dx.doi.org/10.1109/MWC.2007.4407220

[5] J. P. Hespanha, P. Naghshtabrizi and Y. Xu, "A Survey of Recent Results in Networked Control Systems,” Proceedings of the IEEE, Vol. 1, No. 95, 2007, pp. 138-162. http://dx.doi.org/10.1109/JPROC.2006.887288

[6] J. G. Proakis, "Digital Communications,” McGraw-Hill, New York, 1995.

[7] L. Schenato, B. Sinopoli, M. Franceschetti, K. Poolla and S. S. Sastry, "Foundations of Control and Estimation over Lossy Networks,” Proceedings of the IEEE, Vol. 95, No. 1, 2007, pp. 163-187. http://dx.doi.org/10.1109/JPROC.2006.887306

[8] D. M. Zhang and X. G. Wang, "Static Output Feedback Control of Networked Control Systems with Packet Dropout,” International Journal of Systems Science, Vol. 43, No. 4, 2012, pp. 665-672. http://dx.doi.org/10.1080/00207721.2010.517873

[9] R. Buche and H. J. Kushner, "Control of Mobile Communications with Time Varying Channels in Heavy Traffic,” IEEE Transactions on Automatic Control, Vol. 47, No. 6, 2002, pp. 992-1003. http://dx.doi.org/10.1109/TAC.2002.1008363

[10] C. D. Charalambous, S. M. Djouadi and S. Z. Denic, "Stochastic Power Control for Wireless Networks via SDEs: Probabilistic QoS Measures,” IEEE Transactions on Automatic Control, Vol. 51, No. 12, 2005, pp. 43964401.

[11] F. Gunnarsson and F. Gustafsson, "Control Theory Aspects of Power Control in UMTS," Control Engineering Practice, Vol. 11, No. 10, 2003, pp. 1113-1125. http://dx.doi.org/10.1016/S0967-0661(03)00062-5

[12] S. V. Hanly and D.-N. Tse, "Power Control and Capacity of Spread Spectrum Wireless Networks," Automatica, Vol. 35, No. 12, 1999, pp. 1987-2012. http://dx.doi.org/10.1016/S0005-1098(99)00133-8

[13] M. Huang, P. E. Caines and R. P. Malham, "Uplink Po- 
wer Adjustment in Wireless Communication Systems: A Stochastic Control Analysis," IEEE Transactions on Automatic Control, Vol. 49, No. 10, 2004, pp. 1693-1708. http://dx.doi.org/10.1109/TAC.2004.835388

[14] G. Caire, G. Taricco and E. Biglieri, "Optimum Power Control over Fading Channels,” Institute of Electrical and Electronics Engineers, Transactions on Information Theory, Vol. 45, No. 5, 1999, pp. 1468-1489. http://dx.doi.org/10.1109/18.771147

[15] N. A. Pantazis and D. D. Vergados, "A Survey on Power Control Issues in Wireless Sensor Networks," IEEE Communication Surveys and Tutorials, Vol. 9, No. 4, 2007, pp. 86-107. http://dx.doi.org/10.1109/COMST.2007.4444752

[16] P. G. Park, C. Fischione and K. H. Johansson, "Experimental Evaluation of Power Control Algorithms for Wireless Sensor Networks," Proceedings of the IFAC World Congress, Seoul, 2008.

[17] J.-J. Xiao, S. Cui, Z.-Q. Luo and A. J. Goldsmith, "Power Scheduling of Universal Decentralized Estimation in Sensor Networks," IEEE Transactions on Signal Processing, Vol. 54, No. 2, 2006, pp. 413-422. http://dx.doi.org/10.1109/TSP.2005.861898

[18] X. Zhang, H. V. Poor and M. Chiang, "Optimal Power Allocation for Distributed Detection over MIMO Channels in Wireless Sensor Networks," IEEE Transactions on Signal Processing, Vol. 56, No. 9, 2008, pp. 41244140. http://dx.doi.org/10.1109/TSP.2008.924639

[19] D. M. Zhang, X. G. Wang and J. Liu, "Robust Power Control Method for Wireless Sensor Networks with Static Output Feedback," Journal of Control Theory and Application, Vol. 10, No. 1, 2012, pp. 44-49. http://dx.doi.org/10.1007/s11768-012-0035-9

[20] B. Sinopoli, L. Schenato, M. Franceschetti, K. Poolla, M.
I. Jordan and S. S. Sastry, "Kalman Filtering with Intermittent Observations," IEEE Transactions on Automatic Control, Vol. 49, No. 9, 2004, pp. 1453-1464. http://dx.doi.org/10.1109/TAC.2004.834121

[21] X. H. Liu and G. Andrea, "Kalman Filtering with Partial Observation Losses," Proceedings of 43rd IEEE CDC, Bahamas, 14-17 December 2004, pp. 4180-4186.

[22] K. Plarre and F. Bullo, "On Kalman Filtering for Detectable Systems with Intermittent Observations," IEEE Transactions on Automatic Control, Vol. 54, No. 2, 2009, pp. 386-390. http://dx.doi.org/10.1109/TAC.2008.2008347

[23] M. Huang and S. Dey, "Stability of Kalman Filtering with Markovian Packet Losses,” Automatica, Vol. 43, No. 4, 2007, pp. 598-607. http://dx.doi.org/10.1016/j.automatica.2006.10.023

[24] Z. Jin, V. Gupta and R. Murray, "State Estimation over Packet Dropping Networks Using Multiple Description Coding," Automatica, Vol. 42, No. 9, 2006, pp. 14411452. http://dx.doi.org/10.1016/j.automatica.2006.03.020

[25] L. Shi, M. Epstein and R. M. Murray, "Kalman Filtering over a Packet-Dropping Network: A Probabilistic Perspective," IEEE Transactions on Automatic Control, Vol. 55, No. 3, 2010, pp. 594-604. http://dx.doi.org/10.1109/TAC.2009.2039236

[26] W. A. Zhang, G. Feng and L. Yu, "Multi-Rate Distributed Fusion Estimation for Sensor Networks with Packet Losses,” Automatica, Vol. 48, No. 9, 2012, pp. 2016-2028. http://dx.doi.org/10.1016/j.automatica.2012.06.027

[27] E. D. Quevedo, A. Anders, S. L. Alex and D. Subhrakanti, "On Kalman Filtering over Fading Wireless Channels with Controlled Transmission Powers," Automatica, Vol. 48, No. 7, 2012, pp. 1306-1316. http://dx.doi.org/10.1016/j.automatica.2012.03.025 